\title{
REDESCRICCÄO DE ANGIOSTRONGYLUS (PARASTRONGYLUS) COSTARICENSIS ISOLADO DE NOVO HOSPEDEIRO SILVESTRE, PROECHIMYS SP., NA VENEZUELA (METASTRONGYLOIDEA, ANGIOSTRONGYLIDAE)
}

\author{
CLÁUDIA PORTES SANTOS
}

\begin{abstract}
Angiostrongylus (Parastrongylus) costaricensis, nematódeo encon trado em artérias mesentéricas do rato silvestre Proechimys sp., na Venezuela, é redescrito e colocado no subgênero Parastrongylus.

Os Metastrongilídeos, principalmente do gênero Angiostrongylus Kamensky, 1905 são muito controvertidos, e várias ten tativas de reorganização do grupo foram feitas entre outros por Dougherty (1946), Schulz (1951), Skrjabin et al. (1961) e Ash (1967). A partir de 1970, Drózdz analisa as espécies até entẫo estudadas e cria dois novos subgêneros, de acordo com o aspecto dos raios bursais e conforme os grupos zoológicos dos hospedeiros, sendo assim designados:
\end{abstract}

- Angiostrongylus (Angiostrongylus) (= Haemostrongylus Railliet \& Henry, 1907; Cardionema Yamaguti, 1941 e Angiocaulus Schulz, 1951).

- Angiostrongylus (Parastrongylus) ( = Parastrongylus Baylis, 1928; Rodentocaulus Schulz, Orloff \& Kutass, 1933; Pulmonema Chen, 1935 e Rattostrongylus Schulz, 1951).

Kinsella em 1971 apresenta uma chave de classificação onde ainda não considera a existência dos subgêneros propostos por Drózdz. Ainda neste ano é descrita por Morera \& Céspedes a espécie Angiostrongylus costaricensis parasitando o homem.

Já em 1972, Chabaud divide os Angiostrongylinae em oito gêneros distintos, baseado em caracteres evolutivos como tipos de raios bursais, de hospedeiro e sua localizaçāo nos mesmos. No gênero Angiostrongylus considera as mesmas espécies do su bgênero Angiostrongylus (Angiostrongylus), revalida Parastrongylus como gênero e ainda Rodentocaulus. Cria o gênero Morerastrongylus com a espécie tipo $M$. costaricensis, nova combinação para Angiostrongylus costaricensis, que em 1977, Mas-Coma confirma.

Em 1978, Anderson estabelece a família Angiostrongylidae para Angiostrongylinae Böhm \& Gebauer, 1934. Reconsidera A. (Angiostrongylus) Drózdz, 1970\% = Haemostrongylus, Cardionema e Angiocaulus); A. (Parastrongylus) (Baylis, 1928 genus) (= Pulmonema, Rattostrongylus e Morerastrongylus) e revalida Rodentocaulus.

Kotrimavichus \& Delyamure em 1979, provavelmente baseados em Chabaud (1972), referem os gêneros Angiostrongylus, Morerastrongylus, Parastrongylus e Rodentocaulus como pertencentes à subfamília Angiostrongylinae (trabalho nâo obtido).

Esta controvérsia nāo ocorre apenas a nível de gênero. Um estudo comparativo da maioria das espécies pertencentes ao gênero Angiostrongylus demonstra a insu ficiência dos caracteres morfológicos utilizados para descrição de novas espécies. Fica aqui a ressalva, de que muitas destas espécies en traräo em sinonímia se um profundo estudo comparativo dos diversos exemplares for feito.

\section{MATERIAL E MÉTODOS}

Tivemos a oportunidade de examinar o material coletado pelo Prof. Herman Lent em Mérida, na Venezuela em 1973, que nos foi gentilmente cedido. São nematódeos encontrados em artérias mesentéricas do rato silvestre Proechimys sp., sendo três fêmeas e cinco machos, que foram depositados na Coleção Helmintológica do Insti tuto Oswaldo Cruz sob o número 31.665. Os helmintos foram clarificados com ácido acético, fenol ou creosoto de Faia e depois de estudados foram guardados em formol acético.

\section{REDESCRIÇĀO}

\section{Angiostrongylus (Parastrongy/us) costaricensis (Morera \& Céspedes, 1971)}

Macho: Comprimento do corpo: 16,78-18,96 mm; largura do corpo: 0,28 mm; esôfago: 0,25-0,26 mm; espículos: $0,27-0,33 \mathrm{~mm}$. O gu bernáculo não foi observado. Os raios bursais são bem característicos nas extremidades, apresentando dilatações marcantes (Fig. 1). Os raios ventro-ventral e ventro-lateral nascem de um tronco comum, bem separado dos laterais (Fig. 2). Estes, também nascem de um mesmo tronco, que é longo, e se separam quase ao mesmo nível. O raio dorsal externo que se localiza bem perto do tronco lateral, tem aproximadamen te o mesmo tamanho do lateral posterior. $O$ dorsal é curto e apresenta pequenas projeções papilares (Fig. 3). 
Fêmea: Comprimento do corpo: 24,57-27,90 mm; esôfago: 0,22-0,25 mm (Fig. 5); distância da vulva à cauda: 0,21-0,29 mm; distância do ânus à cauda: 0,04-0,06 mm (Fig. 4) e ovos: 0,04 x 0,023-0,027 mm.

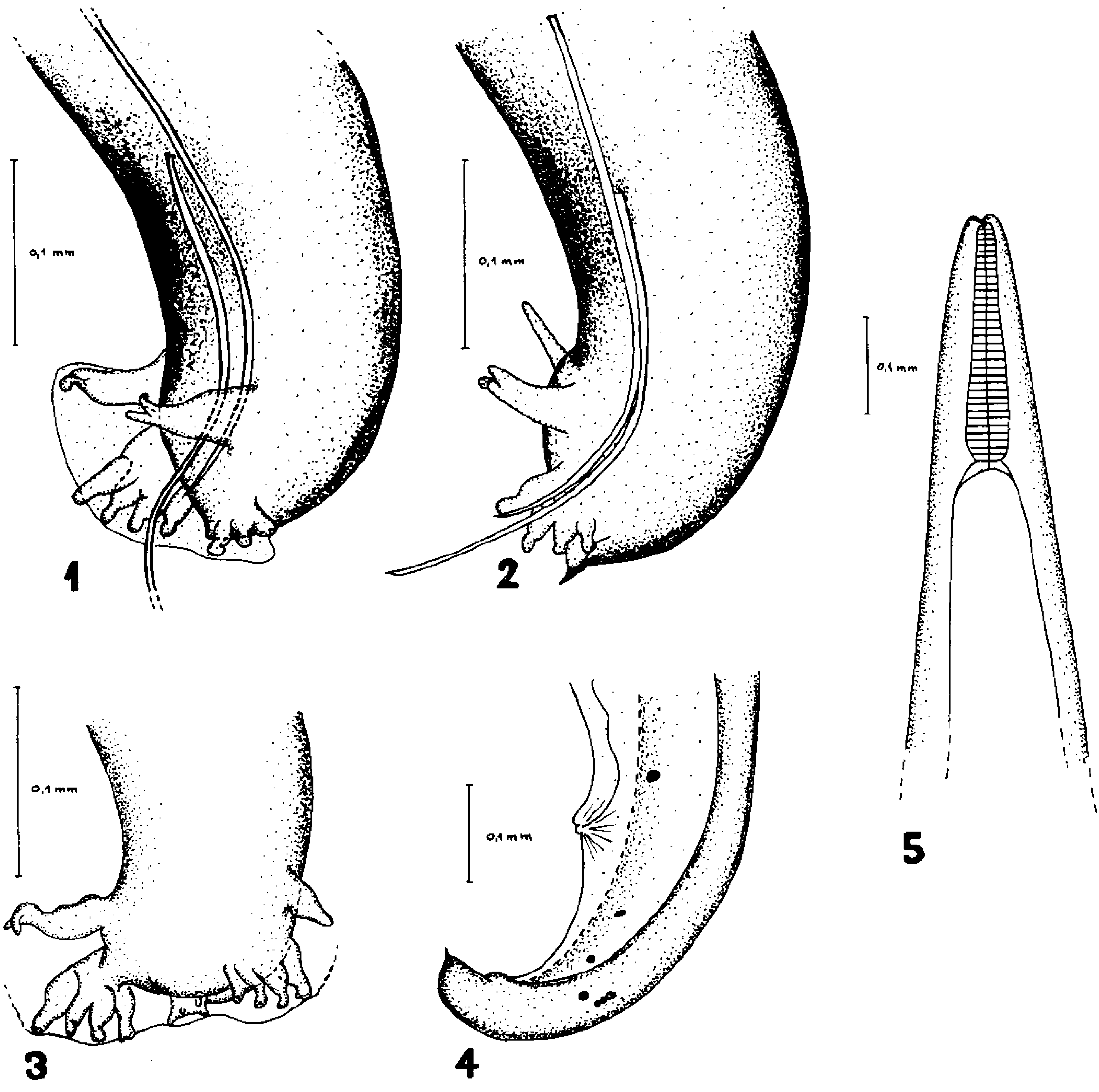

Angiostrongylus (Parastrongylus) costoricensis (Morera \& Céspedes, 1971) - Fig. 1: vista lateral da bolsa copuladora do macho. Fig. 2: cauda do macho com raios bursais e espículos. Fig. 3: vista ventral da bolsa copuladora. Fig. 4: vista la teral da cauda da fêmea apresentando as regiōes anal e vulvar. Fig. 5 : regiāo anterior da fêmea.

\section{COMENTÁRIOS}

Angiostrongylus (Parastrongylus) costaricensis que foi originalmente descrito por Morera \& Céspedes (1971) como Angiostrongylus costaricensis, na verdade deveria tê-lo sido já como pertencen te ao subgênero Parastrongylus, por ser o trabalho de Drózdz an terior à descrição da nova espécie. No próprio trabalho de Drózdz há uma referência a uma nova espécie parasita do homem na Costa Rica.

A caracterização do subgênero Parastrongylus é feita pela bolsa copuladora do macho, que apresenta os raios laterais nascendo de um tronco único longo, e se separando os três praticamente ao mesmo nível. $O$ raio lateral an terior pode algumas vezes se separar primeiro do tronco lateral, mas nunca com um sulco tão profundo como no subgênero Angiostrongylus.

A classificação utilizada por Drózdz e revalidada por Anderson é a mais adequada. Assim, não são válidos os gêneros Morerastrongylus e Parastrongylus, por serem insuficientes a nível de gênero as características apresen tadas, e con tinuam válidos os su bgêneros Parastrongylus e Angiostrongylus. 
A. (P.) costaricensis é um parasito de grande importância médica, por parasitar o homem causando graves problemas. Casos humanos foram referidos na Costa Rica, Honduras, Panamá, Venezuela, México, E.U.A., El Salvador, Brasil, Peru e Colômbia. Apesar de ser um parasi to já de ex tensa distribuiçāo geográfica, muitos médicos dele ainda não têm conhecimento, diagnosticando erroneamente e dificultando um maior conhecimen to das regiōes geográficas parasitadas.

Em trabalhos como os de Wells et al. (1981) e Tesh et al. (1973) os exames foram negativos para Proechimys sp. na Colômbia e Panamá. Assinalamos agora $A .(P$.$) costaricensis como referência em novo$ hospedeiro, Proechimys de espécie não identificada.

\section{SUMMARY}

Angiostrongylus (Parastrongylus) costaricensis is reported from a new host, Proechimys sp., a rodent collected in Venezuela. A study of the species is presented.

\section{REFERÊNCIAS BIBLIOGRÁFICAS}

ANDERSON. R.C., 1978. CIH Keys to the nematode parasites of vertebrates. No. 5. Keys to genera of the Superfamily Metastrongy loidea. Commonweal th Agricultural Bureaux, England.

ASH, L.R., 1967. Angiostrongylus michiganensis sp.n. (Nematoda :Metastrongyloidea), a lungworm occurring in the shrew, Sorex cinereus cinereus, in Michigan. J. Parasit. 53 (3) :625-629.

BAYLIS, H.A., 1928. On a collection of nematodes from Nigerian mammals (chiefly rodents). Parasitology 20:280-304. BOHM, L.K. \& GEBAUER, O., 1934. Zur System der Familie der Metastrongylidae Leiper, 1908. Zool. Anz. 105 (11/12) $: 287-294$.

CHABAUD, A.G., 1972. Description de Stefanskostrongylus dubosti n.sp., parasite du Potamogale et essai de classification des Néma todes Angiostrongylinae. Ann. Parasit. 47 (5):735-744.

CHEN, H.T., 1935. Un nouveau nématode pulmonaire Pulmonema cantonensis, n.g., n.sp., des tats de Canton. Ann. Parasitol. $13(4): 312-317$.

DOUGHERTY, E.C., 1946. The genus Aelurostrongylus Cameron, 1927 (Nematoda:Metastrongylidae) and its relatives, with descriptions of Parafilaroides, gen. nov., and Angiostrongylus gubernaculatus, sp.n. Proc. Helm. Soc. Wash. 13 (1) $: 16-26$.

DRÓZDZ, J., 1970. Révision de la systématique du genre Angiostrongylus Kamensky, 1905 (Nematoda :Metastrongyloidea). Ann. Parasit., 45 (5) :597-603.

KAMENSKY, S.N., 1905. Sistematicheskoe polozhenie radov Metostrongylus worst. i Protostrongylus g.n. sredi drugikh Strongylidae. Sbomik Trudov Khar'kov. Vet. Inst. 7 (2):17-50.

KINSELLA, J.M., 1971. Angiostrongylus schmidti sp.n. (Nematoda: Metastrongyloidea) from the rice rat. Oryzomys palustris, in Florida, with a key to the species of Angiostrongylus Kamensky, 1905. J. Parasit. 57 (3) :494-497.

KOTRIMAVICHUS, V.L. \& DELYAMURE, S.L., 1979. Principles of nematology. Edited by K.M. Ruzhikov V. 29. Filaroididae of Domestic and Wild Animals. $156 \mathrm{pp}$.

MAS-COMA, S., 1977. Gallegostrongylus ibicensis n. gen., n.sp. (Nematoda: Metastrongylidae), parasite pulmonaire de Mus musculus Linnaeus, 1758 (Rodentia: Mundae) à Ibiza (Baleares). Ann. Parasitol. 52 (6) :637-642.

MORERA, P. \& CÉSPEDES, R., 1971. Angiostrongylus costaricensis n.sp. (Nematoda:Metastrongyloidea), a new lungworm occuring in man in Costa Rica. Rev. Biol. Trop. $18(1 / 2): 173-185$.

RAILLIET, A. \& HENRY, A., 1907. Sur les variations des strongyles de l'appareil respiratoire de mammifères. C. R. Soc. Biol. $63(38): 751-753$.

SCHULZ, R.S., 1951. Phylogénèse des nématodes du sous-ordre Strongylata et révision de la systématique de Métastrongyloidea. Doklady A kad. Nauk. SSSR, $80: 293$.

SCHULZ, R.S.; ORLOV, I.V. \& KUTASS, A.J., 1933. Zur Systematik der Subfamilie Syn thetocaulinae Skrj., 1932 nebst Beschreibung einiger neuer Gattungen und Arten. Vorläufige Mitteilung. Zool. Anz. 102 (11/12):303-310.

SKRJABIN, K.I.; SHIKHOBALOVA, N.P.; SCHULZ, R.S.; POPOVA, T.1.; BOEV, S.N. \& DELYAMURE, S.L., 1961. Key to parasitic nematodes, Vol. III. Strongylata, $890 \mathrm{pp}$.

TESH, R.B.; ACKERMAN, L.J.; DIETZ, W.H. \& WILLIAMS, J.A., 1973. Angiostrongylus costaricensis in Panamá. Prevalence and pathologic findings in wild rodents infected with the parasite. Am. J. Trop. Med. Hyg. 22 (3) :348-356.

WELLS, E.A.; D'ALESSANDRO, A.; MORALES, G.A. \& ANGEL, D., 1981. Mammalian wildiffe diseases as hazards to man and livestock in an area of Lianos Orientales of Colombia. J. Wildlife Diseases 17 (1) :153-162.

YAMAGUTI, S., 1941. Studies on the helminth fauna of Japan. Part. 35. Mammalian nematodes II. Japan. J. Zool. 9 (3) $: 409-439$. 